\title{
Cavity optomechanics with photonic bound states in the continuum
}

\author{
Jamie M. Fitzgerald $\odot,{ }^{1,}{ }^{*}$ Sushanth Kini Manjeshwar $\odot,{ }^{2}$ Witlef Wieczorek $\odot,{ }^{2}$ and Philippe Tassin ${ }^{1}$ \\ ${ }^{1}$ Department of Physics, Chalmers University of Technology, SE-412 96 Göteborg, Sweden \\ ${ }^{2}$ Department of Microtechnology and Nanoscience, Chalmers University of Technology, SE-412 96 Göteborg, Sweden
}

(Received 13 July 2020; accepted 14 January 2021; published 10 February 2021)

\begin{abstract}
We propose a versatile, free-space cavity optomechanics platform built from two photonic crystal membranes, one of which is freely suspended, and designed to form a microcavity less than one wavelength long. This cavity features a series of photonic bound states in the continuum that, in principle, trap light forever and can be favorably used together with evanescent coupling for realizing various types of optomechanical couplings, such as linear or quadratic coupling of either dispersive or dissipative type, by tuning the photonic crystal patterning and cavity length. Crucially, this platform allows for a quantum cooperativity exceeding unity in the ultrastrong single-photon coupling regime, surpassing the performance of conventional Fabry-Pérot-based cavity optomechanical devices in the nonresolved sideband regime. This platform allows for exploring new regimes of the optomechanical interaction, in particular in the framework of pulsed and single-photon optomechanics.
\end{abstract}

DOI: 10.1103/PhysRevResearch.3.013131

\section{INTRODUCTION}

Cavity optomechanical devices [1] provide quantum control over their constituent mechanical and optical degrees of freedom for use in precision measurements, quantum networks, and fundamental tests. To this end, optomechanical devices require sufficiently strongly coupled optical and mechanical resonators, along with the minimization of unavoidable decoherence, so they can access the strongcooperativity regime. Experiments have accessed this regime by boosting the optomechanical interaction with a laser drive, resulting in the demonstration of ground-state cooling [2-5], optical [6,7] or mechanical squeezing [8-10], or (opto)mechanical entanglement [11-13]. These experiments have exploited a linear coupling to the mechanical resonator, while nonlinear coupling enables complementary ways to measure and manipulate mechanical motion in the quantum regime [14-19].

Cavity optomechanical platforms can be classified based on whether in-plane or out-of-plane light propagation is used. While in-plane geometries boast the largest coupling rates due to colocalization of photonic and phononic modes [2,2022], they are inherently limited by material loss and structural disorder. The advantage of out-of-plane geometries, such as Fabry-Pérot (FP) cavities in end-mirror [23-25], membranein-the-middle (MiM) [14], or levitated [5] configurations, is that a substantial proportion of light propagation is in vacuum. This leads to lower optical decay rates (one can simply make the photon path length in the cavity longer) but this comes at the price of smaller single-photon coupling rates. In part,

\footnotetext{
*jamief@chalmers.se

Published by the American Physical Society under the terms of the Creative Commons Attribution 4.0 International license. Further distribution of this work must maintain attribution to the author(s) and the published article's title, journal citation, and DOI.
}

the original motivation for the MiM setup was to spatially separate the mechanical and optical functionality, but the resulting weak coupling has naturally led to attempts to increase it. In particular, the concept of multielement optomechanics [26] has been proposed, but its realization is involved [27-30]. Furthermore, as light is trapped in ever smaller volumes, the necessity of the outer cavity becomes questionable [31].

The need in optomechanics for high reflectivity, high mechanical quality factor, and low mass mechanical resonators necessitates a move away from bulky components such as Bragg mirrors, towards ultrathin mirrors. Suspended photonic crystal $(\mathrm{PhC})$ slabs that support guided-mode resonances [32] have been demonstrated to possess over $99.9 \%$ reflectance [33] without compromising on the mechanical properties $[29,34,35]$. In reflection and transmission spectra, the guided mode manifests as an asymmetrical Fano line shape [32]. Placing two PhC slabs close together has long been considered for sensing applications [36-38], and experimental studies have explored placing a single PhC slab in a cavity [33,3942], as well as two PhC slabs in a MiM configuration [29] and as a cavity in their own right [43]. It has only recently become apparent that the internal dynamics of the guided-mode resonance can lead to new optomechanical effects $[44,45]$.

In this work, we propose a platform for cavity optomechanics, constructed from two suspended $\mathrm{PhC}$ slabs in an end-mirror configuration, that relies on photonic bound states in the continuum (BICs). BICs are a general wave phenomenon, where a completely spatially localized mode can exist above the light line [46,47]. The double $\mathrm{PhC}$ slab cavity (DPhoC), depicted in Fig. 1(a), possesses a large optomechanical coupling due to its near-wavelength length and a moderately low decay rate thanks to the near-perfect trapping of light via the BIC. In contrast to conventional end-mirror systems, this simple system can access purely linear dispersive optomechanical coupling via the BIC mechanism, or purely quadratic coupling via evanescent coupling between the slabs. The enhanced flexibility of this platform is due to 

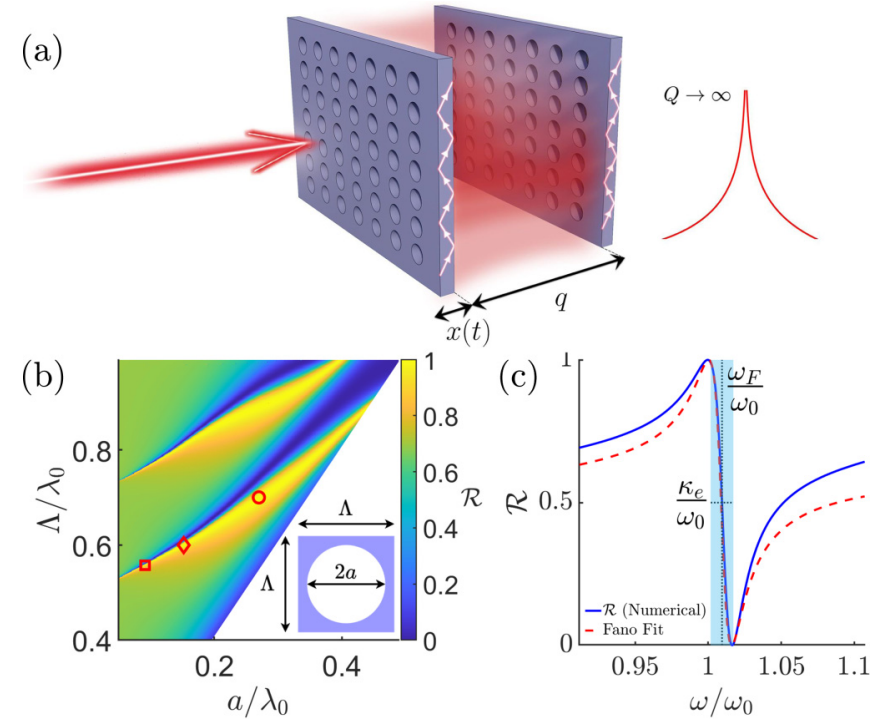

FIG. 1. (a) Illustration of the double-photonic crystal slab cavity (DPhoC) and the bound state in the continuum (BIC) mechanism. (b) Reflectance map of lattice period and air-hole radius for a 100-nm-thick GaAs slab designed to operate at a wavelength $\lambda_{0}=$ $1550 \mathrm{~nm}$. The red markers indicate the three parameter sets used in this work. (c) Reflectance spectrum of a PhC slab (blue line) with $\Lambda=0.6 \lambda_{0}$ and $a=0.1525 \lambda_{0}$, and an exemplary Fano fit (red dashed line).

a complicated interplay of near- and far-field optical coupling between the two slabs. We argue that near-wavelength and subwavelength localization of optical modes is a promising strategy for out-of-plane systems and that the DPhoC, with experimentally realistic parameters, can simultaneously possess the required optical and mechanical properties to access the strong quantum cooperativity regime on the single-photon level, without the encumbrance of an outer FP cavity.

\section{OPTOMECHANICAL COUPLINGS}

For a FP cavity with a movable end mirror, the cavity-mode energy $\hbar \omega_{c}$ depends parametrically on the resonator's out-ofplane displacement $x$ and can be expanded around the equilibrium point $q$, leading to the linear $g_{0}=-\partial \omega_{c} /\left.\partial x\right|_{x=q} x_{0}=$ $G x_{0}$, and quadratic $g_{2}=-\frac{1}{2} \partial^{2} \omega_{c} /\left.\partial x^{2}\right|_{x=q} x_{0}^{2}=G_{2} x_{0}^{2}$, singlephoton coupling rates, where $x_{0}$ is the zero-point motion. We have introduced for convenience the optical frequency shift per displacement $G$ and its counterpart for the second derivative $G_{2}$. Both $g_{0}$ and $g_{2}$ are complex numbers as $\omega_{c}$ is the eigenvalue of an open-cavity problem; the imaginary part gives the decay of the cavity mode $\kappa$. Thus, the real part of the coupling describes dispersive coupling and the imaginary part describes dissipative coupling.

\section{DOUBLE-PHOTONIC CRYSTAL SLAB CAVITY}

Inspired by our recent experimental work [35], the model system is built from 100-nm-thick, GaAs $\mathrm{PhC}$ slabs patterned with a square lattice of circular holes, and designed to operate at a wavelength around $\lambda_{0}=1.55 \mu \mathrm{m}$, where GaAs has a high refractive index of 3.374 [48]. We stress at this point that the

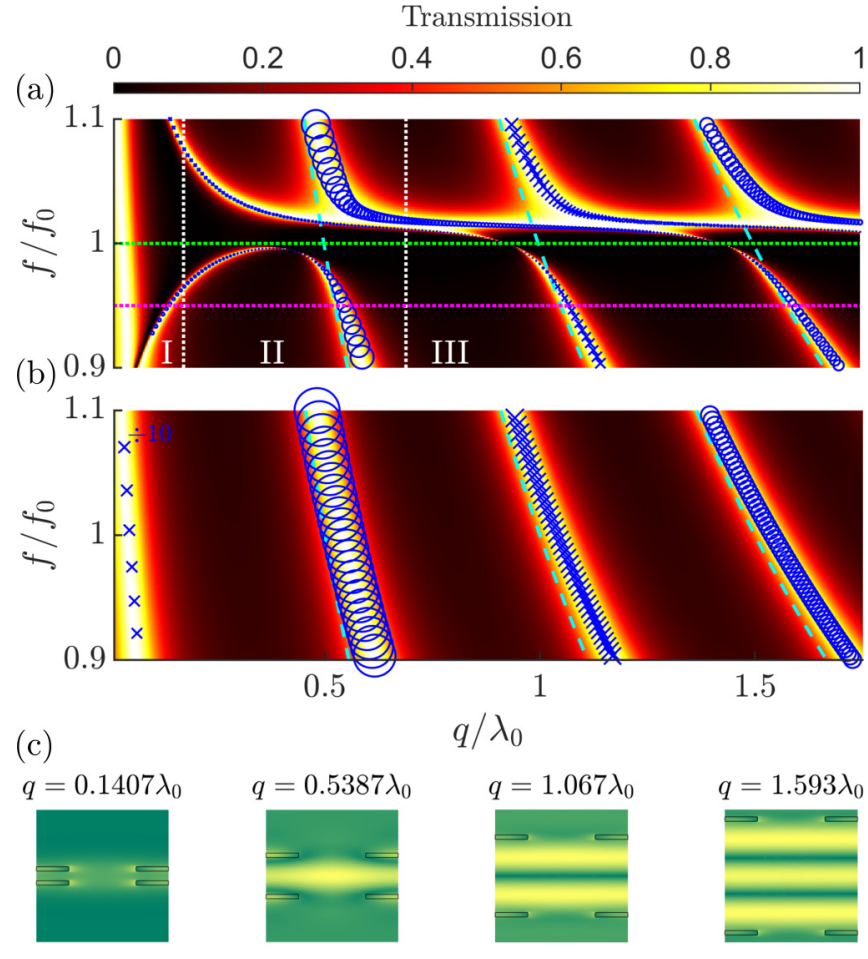

FIG. 2. (a) Transmittance map against frequency and separation for two $\mathrm{PhC}$ slabs with a period of $0.6 \lambda_{0}$ and radius of $0.1525 \lambda_{0}$, with the cavity eigenmodes overlaid in blue. (b) Transmittance map for two homogeneous slabs with an effective refractive index given by the lattice parameters of (a) (see Appendix B for details). (c) Electric field plots for separations corresponding to peak transmittance taken along the slice $f=0.95 f_{0}$ for the DPhoC, as indicated by the magenta dotted line in (a).

physics discussed in this work is not material dependent (see also Appendix F 3) and we expect the same phenomena in, e.g., SiN-based systems [27-29,33,34,49]. To find suitable lattice parameters to achieve a Fano resonance for a single slab, a reflectance map over the air-hole radius and lattice period is calculated and shown in Fig. 1(b). See Appendix A for details on the numerical calculations. The lattice parameters used in this section are indicated by the red diamond marker on Fig. 1(b): a period of $0.6 \lambda_{0}$ and radius of $0.1525 \lambda_{0}$. The reflectance spectrum, depicted in Fig. 1(c), shows a pronounced peak near $\lambda_{0}$, which corresponds to the Fano resonance. The physics behind this is well captured by coupled-mode theory $[32,50]$ and an exemplary Fano fit is plotted in Fig. 1(c), which allows for the extraction of the mode frequency $\omega_{F}$, external decay rate (radiative loss) $\kappa_{e}$, and internal decay rate $\kappa_{i}$ (e.g., due to materials loss). More details on the model are given in Appendix B.

We now consider two such PhC slabs, separated by a distance $q$. The transmittance spectrum is mapped for a range of separations, which is shown in Fig. 2(a). To emphasize that the coupled $\mathrm{PhC}$ slabs do not simply lead to a higher reflectivity, but rather new phenomena, we show in Fig. 2(b) the transmittance map of the corresponding double-homogeneous slab system with an effective refractive index [32]. On top of both transmittance maps, the electromagnetic eigenfrequencies are shown in blue, which are sorted by their even (crosses) or odd 
(circles) symmetry in the $z$ direction. The imaginary part of the eigenfrequency measures the radiative loss of the mode and its value is indicated by the marker size. At this stage, no material loss is included.

The two structured slabs can couple via two possible routes. One is photon tunneling, i.e., a direct evanescent coupling of the slab's near field. The second is an indirect coupling through free-space photon propagation. To aid our discussion, we identify three regions based on the slab separation and the consequent dominant form of interaction between the slabs: near field $\left[q \lesssim \lambda_{0} /\left(2 n_{\text {eff }}\right) \sim 270 \mathrm{~nm}\right]$, intermediate field $\left[\lambda_{0} /\left(2 n_{\text {eff }}\right) \lesssim q \lesssim 2 \lambda_{0} / n_{\text {eff }}\right]$, and far field $(q \gtrsim$ $\left.2 \lambda_{0} / n_{\text {eff }} \sim 1000 \mathrm{~nm}\right)$, separated by white lines in Fig. 2(a). In the far field, the transmittance maps shown in Figs. 2(a) and 2(b) exhibit diagonal bands of high transmittance, which is typical FP behavior. The cavity-mode energies of a perfect FP cavity are indicated by the dashed cyan lines. For a given frequency, the transmittance reaches unity for certain separations where a half-integer number of wavelengths can fit into the cavity [see Fig. 2(c)]. In contrast to the homogeneous slabs, which closely follow the cyan lines, the structured slabs show a much more intricate structure on top of this background. Most pertinent to our discussion is the narrowing of the transmittance bands close to the Fano resonance (indicated by the green dotted line). Further inspection shows that the linewidth of the transmittance spectra approaches zero close to separations corresponding to FP resonances. This behavior is captured by the marker size of the eigenmodes becoming vanishingly small. This is fundamentally different from a conventional FP cavity where the decay rate is inversely proportional to the cavity length; instead, it is indicative of the evolution of the cavity eigenmode into a BIC. Now, the decay rate is given by the nonideality of the $\mathrm{PhC}$ slabs, meaning that the ratio $g_{0} / \kappa$ is dependent on the cavity length. This changes the design philosophy of optomechanic devices based on structured slabs; it is astute to build small cavities to maximize the optomechanical coupling without any increase in the decay rate.

BICs are peculiar resonances that do not decay over time as there are no available radiation channels due to destructive interference; in principle, they have an infinite quality $(Q)$ factor [47]. They have been explored in PhC slabs [51-53] and double-PhC slab structures [54]. In practice, the optical $Q$ factor is limited by structural disorder and material loss; nevertheless, $Q$ factors up to $4.9 \times 10^{5}$ have recently been demonstrated [55]. The BICs we observe here are examples of "resonance-trapped" BICs, where the gap acts as the tunable parameter [47], which are attractive as they are quite robust to imperfections: one need only change the tuning parameter to compensate for geometrical perturbations. An infinite number of BICs exist for the DPhoC for increasing $q$, but occur at ever smaller gradients, indicating a weaker optomechanical coupling. The long lifetime of the photon in the guidedmode resonance allows a moderately low decay rate even for wavelength-sized cavities. This observation is extremely relevant for microcavities for optomechanics; we can boost $g_{0}$ by reducing the cavity length down to $\sim \lambda_{0} / 2$, but with $\kappa$ not limited by the cavity length but rather by the internal loss of the individual slab resonances. A detailed comparison between conventional FP-type optomechanical microcavities and the DPhoC is presented in Appendix E. Despite the huge amount of current interest in BICs [47], there has been limited study of their utility for optomechanics [56,57].

The lowest-order BIC is located in the intermediate-field region, where both coupling via photon tunneling, associated with gradient forces, and propagation, associated with radiation pressure, are relevant. This is illustrated in the electric field plot for $q=0.5387 \lambda_{0}$ in Fig. 2(c) by the deviation from the standard standing-wave mode profile. The series of field profiles show the evolution from near- to far-field dominated interaction between the two slabs as the gap is increased. In the intermediate-field region, we see the same linewidth narrowing due to a BIC in Fig. 2(a), but now the high-transmittance band is highly warped and bends away from the FP line. In contrast to higher-order BICs, the lowestorder BIC is shifted in energy from the individual slabs' Fano resonance (green dotted line) due to the near-field coupling. We also observe the very typical mode splitting of an odd and even mode around the Fano energy. Furthermore, for the lower-energy even mode, there is a crossover from a repulsive to attractive force, i.e., $g$ changes sign. This means that at a certain separation the derivative with respect to displacement vanishes, allowing the DPhoC to access purely quadratic optomechanical coupling: $g_{0}=0, g_{2} \neq 0$. This is in stark contrast to the regular end-mirror configuration, which can only support repulsive forces. The quadratic coupling relies on gradient forces, which depend on the overlap between the near fields of both slabs, and so exhibits an exponential dependence on separation [36,58]: $\zeta \propto \exp (-q / \delta)$, where $\delta$ quantifies the out-of-the-plane decay length of the guided mode. The use of evanescent coupling in optomechanics is nothing new; it has been commonly used to couple light in waveguides to optical microresonators $[59,60]$, as well as microresonators to one another [61], but has rarely been utilized for out-ofplane optomechanics [40,43]. As such, the DPhoC represents a bridge between the two worlds of in-plane and out-of-plane cavity optomechanics: exhibiting both attractive and repulsive optomechanical forces as well as substantial in- and out-ofplane propagation of light. Finally, we note that the near-field zone supports only lossy cavity modes and does not seem suitable for applications in optomechanics; this is discussed further in Appendix D.

All of the physics displayed in the transmittance map in Fig. 2(a) can be captured extremely well by coupled-mode theory $[32,36,50]$, which is detailed in Appendix C. We fit the expressions obtained from coupled-mode theory to the results of numerical simulations to find the value of $\zeta$ and find excellent agreement. More importantly, the theory provides an explanation for the family of BICs we observe. By ignoring the direct reflection and transmission of light through the slab, and considering only the interaction via the excited Fano resonances, we find that the BICs are a predominately far-field phenomena found close to the FP resonances where the cavity decay rate completely vanishes in the absence of internal loss.

\section{ESTIMATED OPTOMECHANICAL COUPLING STRENGTHS}

Since, in principle, a BIC has no radiative loss, its decay rate is given by unavoidable intrinsic loss. To gauge the 

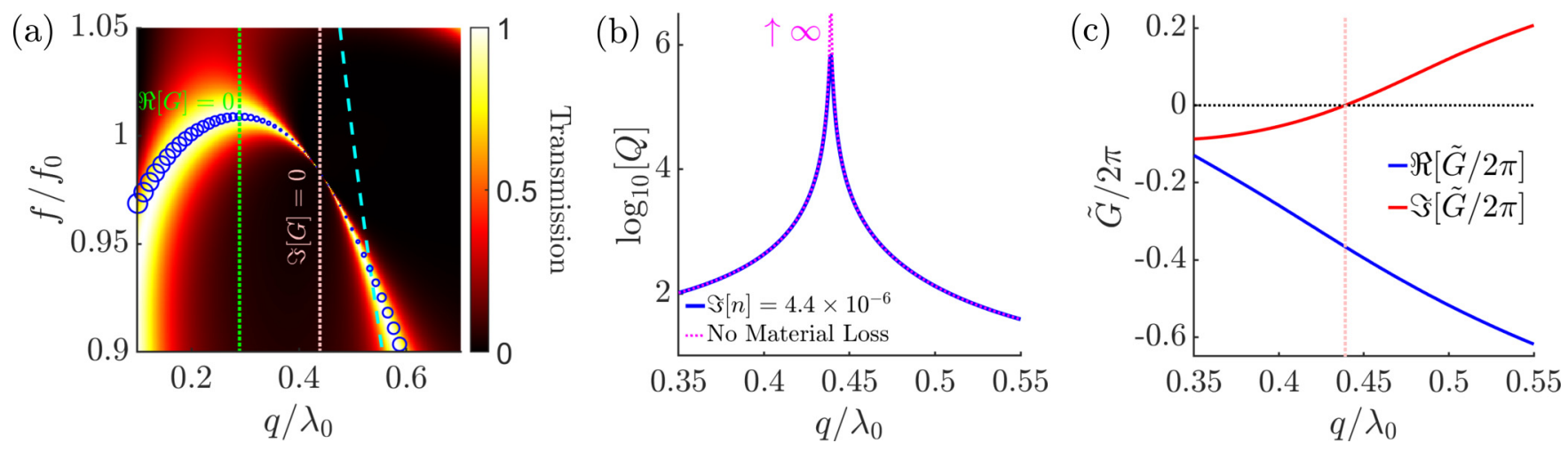

FIG. 3. (a) Transmittance map against frequency and slab separation for a DPhoC with a lattice period of $0.7 \lambda_{0}$ and air-hole radius of $0.27 \lambda_{0}$. (b) Quality factor of the cavity eigenmodes close to the BIC. (c) Dispersive and dissipative parts of the normalized optical frequency shift per displacement: $\tilde{G} / 2 \pi=\frac{d f_{c} / f_{0}}{d q / \lambda_{0}}$. The BIC is indicated by the dotted pink line.

achievable optomechanics performance of the DPhoC, we must estimate the internal loss channels of the PhC slab. In the following, we explore the DPhoC's performance for a set of realistic, albeit challenging, experimental parameters. In Appendix $\mathrm{F} 1$ we also explore a more readily attainable parameter set. We consider intrinsic loss governed by material absorption and use experimental studies of GaAs microdisks [62] to obtain $\operatorname{Im}[n]=4.4 \times 10^{-6}$ (see Appendix F 1 for details). This allows us to estimate the lower bound on the achievable cavity decay rate, assuming that disorder-related loss and finite-size effects of both the beam and the sample can be ignored. We discuss these effects in detail in Appendix F2, where it is shown that the DPhoC can maintain high $Q$ factors over a broad range of wave vectors away from the high-symmetry $\Gamma$ point, making it surprisingly immune from finite-waist and -area effects. Furthermore, in Appendix $G$ we explore practical issues related to geometry and symmetry sensitivity and how they might be tackled in experiments. Finally, we note that ultrashort Fano cavities have been shown to suffer less from finite-waist effects [44], illustrating a further advantage of working with compact cavities using BICs.

\section{A. Dispersive linear coupling}

To highlight the large single-photon cooperativity achievable with the DPhoC, we now change the lattice parameters to boost the dispersive linear coupling at the BIC location: a period of $0.7 \lambda_{0}$, radius of $0.27 \lambda_{0}$, and thickness of $100 \mathrm{~nm}$, indicated by the red circle in Fig. 1(b). This system is very practical, with a double-slab structure very close to these parameters already demonstrated [35]. The transmittance map, shown in Fig. 3(a), exhibits a BIC located in the intermediate zone at $q=0.44 \lambda_{0}$ (pink dotted line), shown explicitly by a sharp peak in the $Q$ factor in Fig. 3(b). The $Q$ factor has a maximum around $Q=\operatorname{Re}\left[f_{c}\right] /(2 \kappa)=6.8 \times 10^{5}$, which is limited by material absorption, and is similar in magnitude with the highest $Q$ factors for a BIC reported to date [55]. In Fig. 3(c) we plot a normalized $G$ and find $G / 2 \pi=-46 \mathrm{GHz} / \mathrm{nm}$ and $\kappa / 2 \pi=\operatorname{Im}\left[f_{c}\right]=140 \mathrm{MHz}$ at the BIC. Because our system is so compact, we can achieve coupling strengths of the order of tens of hundreds of $\mathrm{GHz} / \mathrm{nm}$. This is orders of magnitude larger than conventional out-of-plane systems [14,29] and comparable to values seen for in-plane geometries $[2,20-$
22]. The DPhoC has the advantage that no outer cavity is necessary, as opposed to the MiM geometry [14] or multielement optomechanics approach [26], considerably simplifying fabrication and operation.

The DPhoC has the potential to access the regime of single-photon optomechanics $[63,64]$ by obtaining a large single-photon quantum cooperativity. Using realistic parameters of suspended PhC slabs [34,35] with a mechanical frequency of $\Omega_{m} / 2 \pi=150 \mathrm{kHz}$ and associated effective mass $m_{\text {eff }}=1 \mathrm{ng}$ yields a single-photon optomechanical coupling strength of $g_{0} / 2 \pi=G x_{0} / 2 \pi \sim 3.4 \times 10^{5} \mathrm{~Hz}$ and a considerable $g_{0} / \kappa$ ratio of $\sim 0.0025\left(x_{0}=\sqrt{\hbar / 2 m_{\mathrm{eff}} \Omega_{m}}\right)$. These estimated values place our system in the nonresolved sideband regime and firmly in the ultrastrong single-photon coupling regime with $g_{0} / \Omega_{m} \sim 2.3$, complementing previous works $[21,65]$. Further, assuming a realistically achievable mechanical $Q$ factor of $Q_{m} \sim 10^{8}[34,66]$ yields a singlephoton cooperativity $[1,67]$ of $\mathcal{C}=4 g_{0}^{2} Q_{m} /\left(\kappa \Omega_{m}\right) \sim 2.2 \times$ $10^{6}$, which is similar to Ref. [65] and three orders of magnitude larger than in Refs. [21,22]. When operating the device at moderate cryogenic temperatures $(T=4 \mathrm{~K})$, we predict a remarkable single-photon quantum cooperativity of $\mathcal{C}_{q}=$ $\mathcal{C} / n_{\text {bath }} \sim 4.0\left(n_{\text {bath }}=k_{B} T / \hbar \Omega_{m}\right)$. A value exceeding unity has not been achieved in any cavity optomechanics system before. Thus, the DPhoC offers a promising alternative to proposals in the microwave domain $[68,69]$ or to cavity optomechanics with atoms [70,71]. In these estimations we have assumed uniform mechanical motion, but in reality a mechanical mode will have a spatial profile; this is discussed in Appendix G 3 where additional calculations estimating $m_{\text {eff }}$ for a realistic device are presented.

\section{B. Dissipative linear coupling}

At the BIC separation, the optomechanical coupling is purely dispersive as the cavity decay rate is at a minimum: $\operatorname{Im}\left[g_{0}\right] \propto \partial_{q} \kappa=0$. Isolating purely dissipative coupling is also interesting for certain quantum protocols [72-74]. To this end, we look at the region around $q=0.29 \lambda_{0}$ where $\operatorname{Re}[G] \sim$ 0 , indicated by the green line in Fig. 3(a). Here, the DPhoC exhibits a large dissipative coupling: $\operatorname{Im}[G]=12 \mathrm{GHz} / \mathrm{nm}$. As we are far from the BIC condition, a large decay rate is found. However, dissipative coupling can be utilized for 

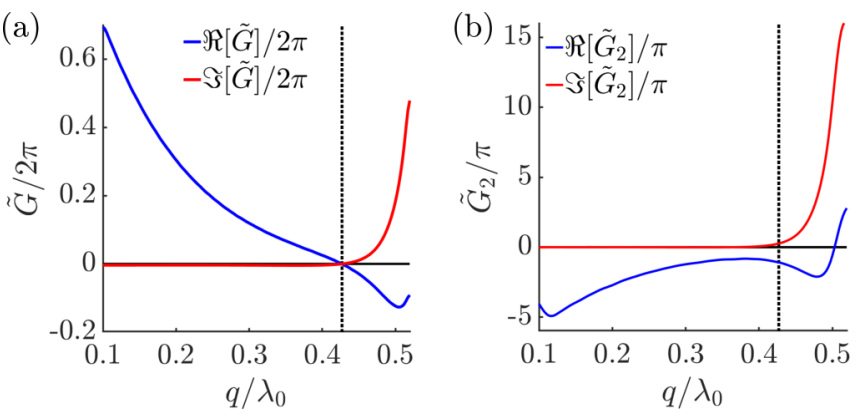

FIG. 4. (a) Dispersive and dissipative parts of $\tilde{G} / 2 \pi$ for a DPhoC with a lattice period $0.5575 \lambda_{0}$ and air-hole radius of $0.092 \lambda_{0}$; this corresponds to the red square in Fig. 1(b). (b) Real and imaginary parts of $\tilde{G}_{2} / 2 \pi=\frac{1}{2} \frac{d^{2} f_{c} / f_{0}}{d q^{2} / \lambda_{0}^{2}}$. The black dotted line gives the location of the BIC at $q=0.43 \lambda_{0}$.

optomechanical cooling without the need for the "good cavity" limit [72].

\section{Quadratic coupling}

Through tuning of the lattice parameters, it is also possible to place the lowest-order BIC at a point of pure quadratic coupling by shifting it to where $\operatorname{Re}[G]$ vanishes [see Fig. 4(a)]. The second derivative $G_{2}$ is shown in Fig. 4(b), illustrating that the quadratic coupling is finite where the linear coupling vanishes. We find a coupling of $\operatorname{Re}\left[G_{2}\right] / \pi=87 \mathrm{MHz} / \mathrm{nm}^{2}$ (and a dissipative coupling of $\operatorname{Im}\left[G_{2}\right] / \pi=6.7 \mathrm{MHz} / \mathrm{nm}^{2}$ ) for a $\kappa / 2 \pi=210 \mathrm{MHz}$. This compares well with the values of $4.5 \rightarrow 30 \mathrm{MHz} / \mathrm{nm}^{2}$ reported by Sankey et al. [15], but the DPhoC has the advantage of being many orders of magnitude more compact, and relies on a different mechanism of evanescent coupling rather than radiation pressure. It remains an open question whether the DPhoC system can be optimized to reach the values of $G_{2} \sim 1 \mathrm{THz} / \mathrm{nm}^{2}$ reported for state-ofthe-art planar $\mathrm{PhC}$ cavities [19].

\section{CONCLUSIONS}

Combining light propagation in both free-space and guided-mode forms, the DPhoC system merges the strengths offered by in-plane and out-of-plane optomechanical systems. We have estimated linear optomechanical coupling rates orders of magnitude larger than conventional endmirror and MiM platforms, at moderately low optical decay rates, potentially leading to a single-photon quantum cooperativity exceeding unity. The DPhoC constitutes a versatile optomechanics platform able to access different regimes of optomechanical coupling that can be used to explore various quantum protocols in the nonresolved sideband regime [4,22,75-77], in particular in the framework of pulsed optomechanics [18,78,79] or frequency-dependent mirrors $[44,45]$. For instance, the strong frequency dependence of the DPhoC's mirrors can be exploited in optomechanical cooling, as recently suggested in Ref. [45]. The geometries described here represent a proof of concept and we expect optimized structures to yield even better performance. We envision many potential pathways from this work, including squeezing of the guided-mode resonance in space using a defect cavity on the PhC slabs to boost photon-phonon colocalization [80], or utilizing phononic BICs [56] alongside their photonic counterparts.

\section{ACKNOWLEDGMENTS}

We acknowledge support from Chalmers' Excellence Initiative Nano, the Swedish Research Council under Projects No. 2016-03603 and No. 2019-04946, and the QuantERA project C'MON-QSENS. Some of the numerical calculations were performed on resources provided by the Swedish National Infrastructure for Computing at C3SE. W.W. acknowledges support from the Knut and Alice Wallenberg foundation through a Wallenberg Academy fellowship.

\section{APPENDIX A: NUMERICAL METHODS}

The numerical calculations are a combination of simulations based on the finite-element frequency domain method (using COMSOL MULTIPHYSICS) and rigorous coupled-wave analysis (RCWA) (using the $S^{4}$ code [81]). Where possible, results were obtained using both methods and excellent agreement to within a few percent was found.

\section{APPENDIX B: FANO RESONANCE}

The interference between the direct transmission of light and the guided mode of a structured slab leads to unity reflection near the guided-mode resonance $\omega_{F}$, with a width $\kappa_{e}$. Due to the large $Q$ factor of the underlying guided modes, Fano resonances are well described by coupled-mode theory (CMT) applied to a single resonator with two ports [32,50]. Both $\omega_{F}$ and $\kappa_{e}$ can be found by calculating the reflection or transmission spectrum using numerical techniques to solve Maxwell's equations and fitting the following expressions:

$$
\begin{gathered}
r(\omega)=\frac{r_{d}\left(\omega-\omega_{F}\right)+t_{d} \kappa_{e}}{\left(\omega-\omega_{F}\right)+i \kappa_{e}}, \\
i t(\omega)=\frac{-i r_{d} \kappa_{e}+i t_{d}\left(\omega-\omega_{F}\right)}{\left(\omega-\omega_{F}\right)+i \kappa_{e}},
\end{gathered}
$$

which are derived under the assumption that the system possesses time-reversal symmetry, conservation of energy, and even symmetry with respect to the mirror plane. $r_{d}$ and $t_{d}$ are given by the reflectivity and transmission of a homogeneous slab with an effective refractive index [32]. For a structured slab with air holes of radius $a$ and period $\Lambda$, the effective index is given by $n_{\mathrm{eff}}=(1-\eta) n+\eta$ where $\eta=\pi a^{2} / \Lambda^{2}$. An example of the fit is shown by the red dashed line in Fig. 1(c). The radiative decay is quantified by $\kappa_{e}$ and linked to the width of the Fano line shape (given by the shaded blue region in the plot). It describes the in and out coupling of the guided mode to external radiative channels. The inverse of $\kappa_{e}$ gives the typical travel time of a photon within the slab. Smaller air holes lead to a reduced $\kappa_{e}$ due to decreased scattering of the in-plane light, but this comes at the price of a larger impact from internal loss [82], denoted by $\kappa_{i}$, which includes the impacts of various loss channels such as material loss and lattice imperfections. 


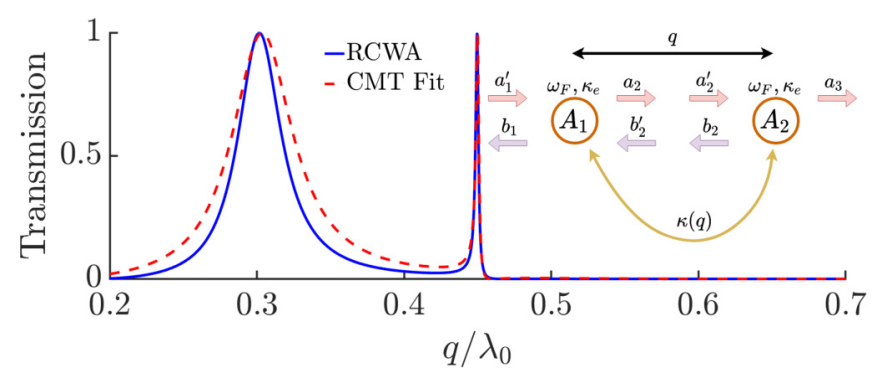

FIG. 5. Slice of the transmittance for $f=0.99 f_{0}$ calculated with RCWA (blue line) for a DPhoC with 100-nm-thick slabs, $\Lambda=0.7 \lambda_{0}$ and $a=0.27 \lambda_{0}$. A fit of the coupled-mode theory (CMT) (red dashed line) is shown, along with an inset illustrating the CMT.

\section{APPENDIX C: BOUND STATE IN THE CONTINUUM THEORY}

The DPhoC is modeled as two resonators within CMT, $A_{1}$ and $A_{2}$, which obey the following two coupled first-order differential equations [36]:

$$
\begin{aligned}
\partial_{t} A_{1}(t)= & \left(-i \omega_{F}-\kappa_{e}\right) A_{1}(t) \\
& +\sqrt{-\kappa_{e}\left(r_{d}+i t_{d}\right)}\left(a_{1}+b_{2} e^{i k q}\right)+i \zeta A_{2}(t), \\
\partial_{t} A_{2}(t)= & \left(-i \omega_{F}-\kappa_{e}\right) A_{2}(t) \\
& +\sqrt{-\kappa_{e}\left(r_{d}+i t_{d}\right)} a_{2} e^{i k q}+i \zeta A_{1}(t),
\end{aligned}
$$

where evanescent coupling is described by the real parameter $\zeta=C e^{-q / \delta}$ and coupling via photon propagation is described by the complex term $e^{i k q} . a_{1}, b_{1}, a_{2}, b_{2}$, and $a_{3}$ are the incoming and outgoing field amplitudes on either side of the slabs, and are defined in the inset of Fig. 5. The transmission it $=a_{3} / a_{1}$ can be found by Fourier transforming, and the remaining parameter $\zeta$ is found by fitting the spectrum for fixed frequency and variable $q$. An example of this fitting procedure is shown in Fig. 5 by the red dashed line. The fit is excellent, showing that this simple model captures both nearand far-field coupling between the slabs.

To illustrate why BICs occur for the DPhoC, we will make some drastic simplifications to the CMT that reveal the essential mechanisms more clearly. The physics we are interested in depends on the interaction of the resonances in each slab and not the direct process which is controlled by $r_{d}$ and $t_{d}$, therefore, we set $r_{d}=0$ and $i t_{d}=1$. This "flat-background" approximation is most valid for PhC slabs with large air holes and, hence, a lower effective refractive index. The coupling of the two resonator modes leads to hybridization into even and odd "supermodes" [83]:

$$
A_{\text {even } / \text { odd }}(t)=\frac{A_{1}(t) \pm A_{2}(t)}{\sqrt{2}},
$$

which have the following energies and decay rates:

$$
\begin{gathered}
\omega_{\text {even/odd }}=\omega_{F} \mp\left[\zeta(q)-\kappa_{e} \sin \left(k_{0} q\right)\right], \\
\gamma_{\text {even/odd }}=\kappa_{e}+\kappa_{i} \pm \kappa_{e} \cos \left(k_{0} q\right),
\end{gathered}
$$

where we have made the approximation that the right-hand side can be evaluated at the Fano energy $\omega_{F}=c k_{0}$. The mode frequency shows a splitting between the even (which is at a

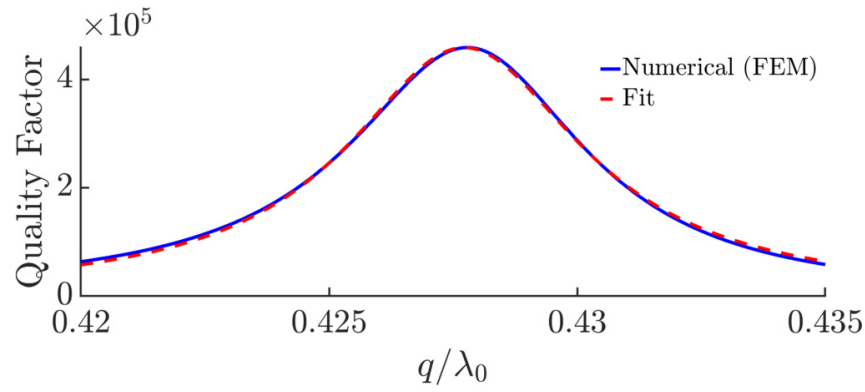

FIG. 6. Quality factor of the cavity modes around a BIC for two PhC slabs of 100-nm thickness, period of $0.5575 \lambda_{0}$ and $a=0.092 \lambda_{0}$. Shown also with a Lorentzian fit $Q_{\text {fit }}(q)=C^{2} /\left[\left(q-q_{0}\right)^{2}\right]$, where $C=1.9809 \lambda_{0}^{2}$ and $q_{0}=0.43 \lambda_{0}$.

lower energy) and the odd mode about the Fano energy, with contributions from both the near-field and far-field coupling, as is observed in Fig. 2(a). These equations also reveal the presence of BICs: for no internal loss, coupling to output channels vanishes for $\cos \left(k_{0} q\right)=\mp 1$, which is just the usual FP resonance condition and reveals an infinite number of such BICs. Equation (C3) explains why the lowest-order BIC is shifted in energy away from the individual slabs' Fano resonance [green dotted line in Fig. 2(a)] by the near-field interaction, while the higher-order BICs at larger gaps occur almost exactly at this energy.

Further evidence that we are indeed observing BICs comes from the quadratic dependence of $1 / Q$ on $q-q_{0}$, where $q_{0}$ is the slab separation corresponding to a BIC [84]. This is confirmed by fitting $Q\left(q-q_{0}\right)$ and is shown in Fig. 6.

\section{APPENDIX D: NEAR-FIELD REGION}

Here, we discuss the near-field region shown in Figs. 2(a) and 2(b), where photon propagation between the slabs is negligible and evanescent coupling dominates. For the structured slabs, this region is indicated by the eigenmodes deviating from the bands of high transmittance. The eigenmodes become very lossy $(Q \sim 10)$ and so are not shown in Fig. 2(a) for clarity. These modes could be useful for cavity optomechanics if we borrow the MiM philosophy and the DPhoC was placed within a larger cavity to recycle the leaked light.

We also observe an interesting high-transmittance branch for the homogeneous slabs in Fig. 2(b). It derives from a family of leaky modes which do not correspond to FP modes. This is illustrated nicely in Fig. 7, where the electric field profile of the lowest-order FP mode and the near-field zone mode are compared; the field of the former is concentrated within the cavity between the slabs, and the field of the latter is concentrated much more within the slabs. As the slabs are not structured and the incoming light is normally incident, it cannot be a consequence of near-field coupling and instead we speculate that it is similar in nature to zero-frequency modes seen for single slabs $[85,86]$.

Note that we do not include Casimir forces, which are derived from vacuum quantum fluctuations and are not present in our classical calculations. 

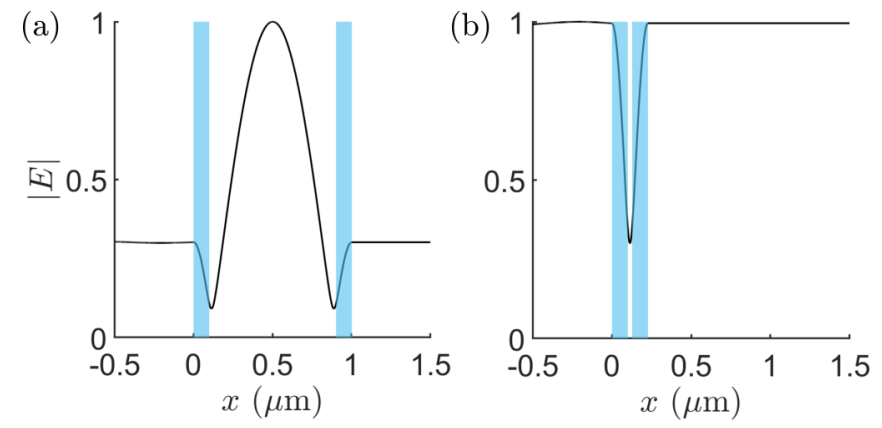

FIG. 7. (a) The lowest-order Fabry-Pérot electric field profile and (b) the high-transmittance near-field mode. Calculated using the transfer-matrix method for incident light from the left-hand side at a wavelength of $1550 \mathrm{~nm}$.

\section{APPENDIX E: COMPARISON OF THE DPhoC TO A FABRY-PÉROT-TYPE OPTOMECHANICAL MICROCAVITY}

For the end-mirror configuration of length $q$, the linear dispersive coupling rate is given by $\operatorname{Re}\left[g_{0}\right]=\omega_{c} x_{0} / q$, where $x_{0}=\sqrt{\hbar / 2 m_{\text {eff }} \Omega_{m}}$ is the zero-point motion and $\omega_{c}$ is the cavity frequency. For the MiM geometry, the maximum linear coupling rate is $2|r|$ times larger than the corresponding endmirror geometry of the same total cavity length, where $|r|$ is the reflectivity of the inner membrane. In principle, $g_{0}$ can be increased as we decrease the length down to $q=\lambda / 2$ (below which no FP resonance is supported). The decay rate of an end-mirror cavity is given by $\kappa_{c}=\frac{\pi c}{2 q \mathcal{F}}$, where $\mathcal{F}$ is the cavity finesse. This means that $\operatorname{Re}\left[g_{0}\right] / \kappa_{c}$ is independent of length. This is in contrast to the DPhoC where the decay rate is given by the nonideality of the PhC slabs and means that the ratio $\operatorname{Re}\left[g_{0}\right] / \kappa$ is dependent on length.

Let us estimate the optomechanical parameter regime achievable with a FP-type optomechanical microcavity, which in turn allows us to compare to the performance of the DPhoC. To this end, we combine parameters from independent realizations of state-of-the-art optical microcavities [87] and distributed Bragg reflector (DBR) based high-reflectivity mechanical resonators [88-90] in order to obtain an estimate on the potential of a FP-based optomechanical microcavity. We consider an optical microcavity of length $q=17 \mu \mathrm{m}$ with a finesse of $\mathcal{F}=5 \times 10^{5}$ at telecom wavelengths, which has been recently realized in chip-based silicon microcavity arrays [87]. Note that a slightly smaller finesse of $1.8 \times 10^{5}$ has been achieved in a 5-cm-long FP-based optomechanical system [90]. Both of these cavities employed multilayer coatings, i.e., DBRs, to achieve such an exceptionally large finesse. Hence, the mechanical resonator has to be realized via a suspended DBR [89] or a DBR on a mechanical resonator
$[88,90]$ to obtain such high-finesse values. These systems have typical mechanical parameters of $\Omega_{m} / 2 \pi \sim 500 \mathrm{kHz}, m_{\mathrm{eff}} \sim$ $40 \mathrm{ng}$ and a mechanical quality factor $Q_{m} \sim 10^{6}$ at $T \sim 4 \mathrm{~K}$ [88-90]. Note that the DBR limits the performance of the mechanical resonator, in particular, resulting in a lower mechanical quality factor and larger effective mass compared to state-of-the-art DBR-free mechanical resonators, which routinely achieve values of $m_{\text {eff }} \sim 1 \mathrm{ng}, Q_{m}>10^{8}$. All together, this leads to the set of parameters displayed in Table I and, hence, to much less advantageous optomechanical values than the DPhoC we propose in this work, with the exception of a slightly improved $\kappa / \Omega_{m}$ ratio.

Also shown in Table I are the parameters for the minimal cavity length of $q=\lambda_{0} / 2$ of such a hypothetical FP cavity. Despite this microcavity having a larger $G$ than the DPhoC we consider, such a system suffers from the ratio $g_{0} / \kappa$ being independent of cavity length, and a worse performance of the mechanical resonator compared to PhC-based mechanical resonators. In particular, the single-photon quantum cooperativity of both conventional FP cavities considered in Table I are orders of magnitude smaller than one.

\section{APPENDIX F: ESTIMATION OF LOSS CHANNELS}

\section{Material absorption loss}

To estimate the ultimate upper bounds on the BIC's $Q$ factor, we need an estimate of the intrinsic material loss. We extracted material-based absorption for GaAs using Ref. [62], where a loss rate of $\frac{\kappa_{i}}{2 \pi} \sim 0.5 \mathrm{GHz}$ was measured for GaAs microdisks at $1600 \mathrm{~nm}$. This yields an absorption coefficient $\alpha=\kappa_{i} / v_{g} \sim 0.3 \mathrm{~cm}^{-1}$ with the group velocity $v_{g}$ estimated as $\sim 10^{8} \mathrm{~m} / \mathrm{s}$. For the 100 -nm-thick membranes we consider in this work, we get a material absorption of about $3 \mathrm{ppm}$; this is an overestimation of the loss as some of the electric field of the mode will be concentrated in the air holes rather than the GaAs. The imaginary component of the refractive index can then be found from $\operatorname{Im}[n]=\frac{\alpha \lambda_{0}}{4 \pi}$ [91]. For our operation wavelength of $\lambda_{0}=1550 \mathrm{~nm}$, this gives $\operatorname{Im}[n] \sim 4.4 \times 10^{-6}$. This value, along with a mechanical quality factor of $Q_{m}=10^{8}$, will be denoted as parameter set I and displayed in Table II. This set was used in the main text and represents challenging, but achievable, parameters that are state of the art in both mechanics and photonics. Using this value of $\operatorname{Im}[n]$ for a single $\mathrm{PhC}$ slab gives a max reflectance of $R=0.99998$ near the Fano resonance.

For set II, we estimate the corresponding effective $\operatorname{Im}[n]$ for a maximum reflectance of 0.999 , which was achieved in a single $\mathrm{Si}_{3} \mathrm{~N}_{4} \mathrm{PhC}$ slab in Ref. [33]. This yields $\operatorname{Im}[n]=$ $2 \times 10^{-4}$. This is not entirely appropriate as the devices in the aforementioned reference were limited by scattering rather than material absorption, but it gives an indication of the ef-

TABLE I. Optomechanical parameters for optical microcavities of length $17 \mu \mathrm{m}$ and $\lambda_{0} / 2$. Common parameters between both sets are a finesse of $500000, m_{\mathrm{eff}}=40 \mathrm{ng}, \Omega_{m} / 2 \pi=500 \mathrm{kHz}$, and $Q_{m}=10^{6}$ at $T=4 \mathrm{~K}$.

\begin{tabular}{ccccccccc}
\hline \hline$L$ & $\kappa / 2 \pi(\mathrm{MHz})$ & $G / 2 \pi(\mathrm{GHz} / \mathrm{nm})$ & $g_{0} / 2 \pi(\mathrm{kHz})$ & $g_{0} / \kappa$ & $g_{0} / \Omega_{m}$ & $\kappa / \Omega_{m}$ & $\mathcal{C}$ & $\mathcal{C}_{q}$ \\
\hline $17 \mu \mathrm{m}$ & 8.8 & 11.4 & 7.3 & $8.3 \times 10^{-4}$ & $1.5 \times 10^{-2}$ & 18 & 49 & $2.9 \times 10^{-4}$ \\
$775 \mathrm{~nm}$ & 193 & 250 & 161 & $8.3 \times 10^{-4}$ & 0.32 & 390 & 1100 & $6.4 \times 10^{-3}$ \\
\hline \hline
\end{tabular}


TABLE II. Optomechanical parameters for linear dispersive coupling for the DPhoC. For both parameter sets $\Omega_{m} / 2 \pi=150 \mathrm{kHz}, G / 2 \pi=$ $-46 \mathrm{GHz} / \mathrm{nm}$, and $m=1 \mathrm{ng}$.

\begin{tabular}{llllllll}
\hline \hline Set & $Q_{m}$ & $\operatorname{Im}[n]$ & $R$ & $\kappa / 2 \pi(\mathrm{MHz})$ & $g_{0} / \kappa$ & $\mathcal{C}$ & $\mathcal{C}_{q}$ \\
\hline I & $10^{8}$ & $4.4 \times 10^{-6}$ & 0.99998 & 140 & 0.0025 & $2.2 \times 10^{6}$ \\
II & $10^{7}$ & $2 \times 10^{-4}$ & 0.999 & 6200 & $5.5 \times 10^{-5}$ & 5000 \\
\hline \hline
\end{tabular}

fects of nonunity reflectance and the resulting optical $Q$ factor of $\sim 10^{4}$ is in line with values found for typical BIC systems [92]. The parameters for set II are also shown in Table II.

\section{Transverse effects}

In our estimation of $g_{0}$, we have ignored loss from transverse effects such as wavefront curvature, non-perfectlyparallel mirrors, and finite-area structures. These unavoidable limitations are a consequence of incident light coupling into modes located over a finite region of $k$ space, leading to additional loss channels. Relevant to our discussions is that ultrashort cavities built from Fano mirrors have been shown to suffer less from finite-waist effects [44]. There is also the possibility of designing Fano mirrors with focusing abilities [93,94]. Furthermore, resonance-trapped BICs have been shown to display a large $Q$ factor over a wide range in $k$ space [53], and recently the merging of multiple BICs has been used to suppress out-of-plane scattering losses [55].

To demonstrate that the DPhoC is surprisingly immune from finite-size effects, we have calculated the $Q$ factor for wave vectors away from the high-symmetry $\Gamma$ point of the first Brillouin zone for a square lattice (see Fig. 8). To save simulation time, we explore slices in $k$ space in the direction from $\Gamma$ to the other high-symmetry points $X$ and $M$. A detailed calculation would integrate over a specified area of $k$ space (a thorough discussion of including finite-beam-waist size effects in reflection and transmission spectra can be found in the Supplemental Material of Ref. [35]). We observe that the mode at $\lambda_{0}$ is doubly degenerate at the $\Gamma$ point and splits in

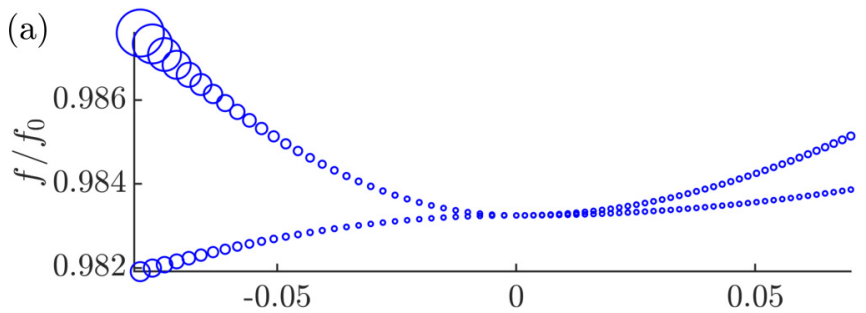

(b)

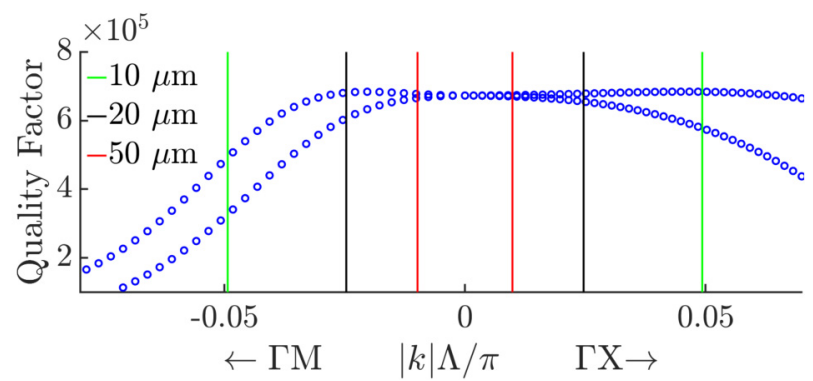

FIG. 8. (a) Energy splitting and (b) quality factor of the DPhoC eigenmodes around the $\Gamma$ point. DPhoC parameters are lattice period of $0.7 \lambda_{0}$, air-hole radius of $0.27 \lambda_{0}$, and slab separation of $0.439 \lambda_{0}$. energy, which can be seen in Fig. 8(a). Importantly, the $Q$ factor remains well above $10^{5}$ in a large region of $k$ space; this is shown in Fig. 8(b). In realistic devices, there is a compromise between the lateral size of the device, which will affect mechanical properties, and the beam-waist size to achieve maximum slab reflectivities. A Gaussian beam can be represented as a sum of angled plane-wave components weighted by a Gaussian distribution with a standard deviation given by the beam divergence $\theta_{0}=\lambda /\left(\pi w_{0}\right)$, where $w_{0}$ is the beam-waist size. In Fig. 8(b), we represent with colored lines the beam divergence for beam waists of 10,20 and $50 \mu \mathrm{m}$, which are typical values used in experiments [29,34,95].

A finite structure, of characteristic length $L$, will support BICs with a finite $k$-space mode profile of $\sim \pi / L$. While a detailed exploration of this is beyond the scope of this work, using Fig. 8(b) allows us to estimate that we need a device with an area much larger than $\sim 20 \times 20 \mu \mathrm{m}^{2}$ to achieve an optical $Q$ factor well above $10^{5}$ (this is estimated by taking a $k$ span of $\frac{\delta k \Lambda}{\pi}=0.05$, which corresponds to $Q$ factors above $2 \times 10^{5}$ ). This is a smaller area than the $\mathrm{PhC}$ slabs reported in our recent experimental work of $\sim 50 \times 50 \mu \mathrm{m}^{2}$ [35]. There exist methods to counter finite-size effects such as using BICs with ultraflat dispersion [96], which could be implemented in future work.

\section{Estimate for SiN-based system}

The physics discussed in this work is not material dependent and can be expected to be applicable for SiN-based systems [27-29,33,34,49], which are more commonly used in optomechanics. Here, we provide an estimate of the optomechanical parameters obtainable with such systems. The optical $Q$ factor of the BIC can be approximated using [91]

$$
\frac{1}{Q_{\mathrm{BIC}}}=\frac{1}{Q_{\mathrm{abs}}} \sim \frac{2 \operatorname{Im}[n]}{\operatorname{Re}[n]},
$$

which we have confirmed to be an accurate estimate for GaAs PhC slabs. $\operatorname{Im}[n]$ as small as $2 \times 10^{-6}$ has been measured for membranes of close to 100-nm thickness at a wavelength of $1064 \mathrm{~nm}$ [97]. As $\operatorname{Re}[n]=2.021$, this gives $Q_{\mathrm{BIC}} \sim 5 \times 10^{5}$. Assuming similar optomechanical coupling rates and mechanical properties, then the ultimate achievable optomechanical parameters of a SiN-based DPhoC should be similar to set I in Table II.

\section{APPENDIX G: EXPERIMENTAL REALIZATION}

\section{Gap sensitivity}

Gap sensitivity is an important aspect to consider for experimental demonstration of the DPhoC. To explore this we take the exemplary DPhoC presented in Fig. 3 (lattice period of $0.7 \lambda_{0}$ and air-hole radius of $0.27 \lambda_{0}$ ) and find the range of gaps 

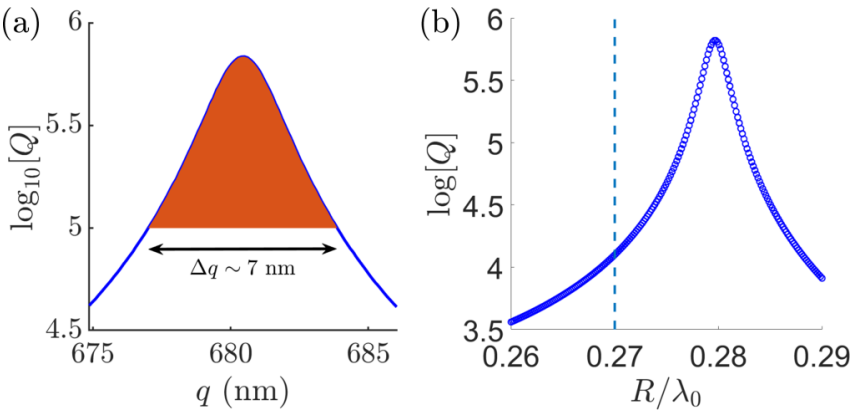

FIG. 9. (a) The $Q$ factor of the DPhoC of period $0.7 \lambda_{0}$ and radius $R_{0}=0.27 \lambda_{0}$ for varying gap size, the operation region corresponding to $Q$ factors over $10^{5}$ is indicated by the orange area and the doubleheaded arrow. (b) The $Q$ factor for a DPhoC of period $0.7 \lambda_{0}$ and gap $q_{\mathrm{BIC}}-10 \mathrm{~nm} \approx 670 \mathrm{~nm}$ for varying radius. The blue dashed line indicates the radius corresponding to the original BIC at $q_{\text {BIC. }}$.

that give a near-BIC mode $Q$ factor over $10^{5}$ [see Fig. 9(a)]. We find an operating range of approximately $7 \mathrm{~nm}$, which is achievable with typical MBE-grown material as, for example, discussed in Ref. [35].

We propose that one could fabricate slabs of different $\mathrm{PhC}$ pattern parameters for the same gap, as the BIC exists for a continuous range of $\mathrm{PhC}$ pattern parameters. To this end, we have performed some further simulations to explore this: we have taken a DPhoC system, again based on Fig. 3, and fixed the gap to $670 \mathrm{~nm}$, this is $10 \mathrm{~nm}$ smaller than the gap corresponding to the BIC $\left(q_{\mathrm{BIC}}=0.439 \lambda_{0} \approx 680 \mathrm{~nm}\right)$. We now vary the air-hole radius in each slab and plot the $Q$ factor of the near-BIC modes [see Fig. 9(b)]. The BIC is simply shifted to a larger radius and we can conclude that by building devices with different radii one could realize the BIC in case the gap is not the one intended.

Furthermore, in situ tuning may be possible via the piezoelectric effect when using piezoelectric materials for realizing the DPhoC. For instance, an AlGaAs spacer in-between the two GaAs slabs could be used to control the gap via an applied voltage. The piezoelectric coefficient of $\mathrm{GaAs}$ is $-2.7 \times 10^{-12} \mathrm{~m} / \mathrm{V}$ and $(-2.7-1.13 x) \times 10^{-12} \mathrm{~m} / \mathrm{V}$ for $\mathrm{Al}_{x} \mathrm{Ga}_{1-x}$ As [98], this would allow for sub-nm level tuning for the gap. It may even allow for the tuning of the $\mathrm{PhC}$ air-hole radius as well.

\section{Symmetry sensitivity}

It is also interesting to explore how precisely the two $\mathrm{PhC}$ slabs must be geometrically matched. As the lattice constant will be practically identical in each slab for the fabrication approach we employed in our recent experimental work [35], we have performed additional eigenmode calculations exploring asymmetry by changing the air-hole radius of one of the slabs, while keeping all other parameters constant. In Fig. 10(a) a plot of the optical $Q$ factor of the near-BIC mode is shown as a function of the difference in radius $\Delta R=R_{1}-R_{0}$, which is given in units of the experimental uncertainty in radius $\left(\delta_{R}=\right.$ $1.8 \mathrm{~nm}$ ) from Ref. [35]. We find that high-quality factors well over $10^{5}$ remain up to one standard deviation.

To explore if geometric mismatch can be compensated by adjustment of other geometrical parameters, we have per-
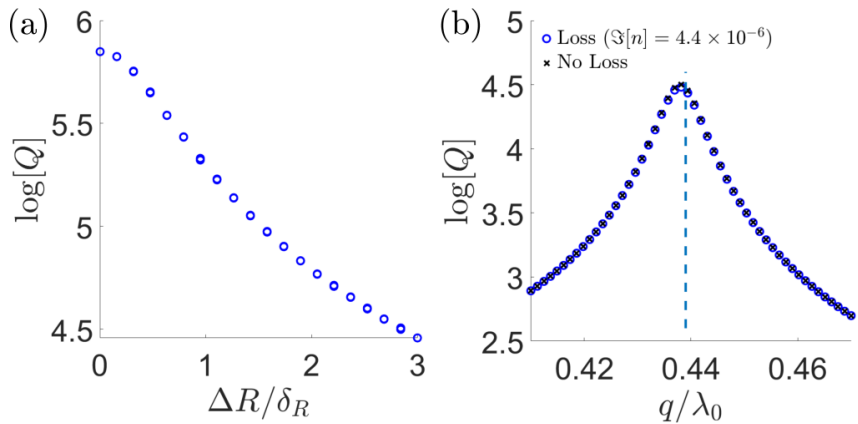

FIG. 10. (a) $Q$ factor of a near-BIC mode as the radius in one of the PhC slabs in a DPhoC is varied for fixed parameters: $\Lambda=0.7 \lambda_{0}$, $q=0.439 \lambda_{0}$ and unmodified radius $R_{0}=0.27 \lambda_{0}$. The difference in radius $\Delta R$ is given in units of the experimental uncertainty $\delta_{R}=1.8$ nm. (b) $Q$ factor of the near-BIC mode as the gap is varied for a DPhoC of $\Lambda=0.7 \lambda_{0}$ and radii of $R_{0}$ and $R_{0}+3 \delta_{R}$.

formed an eigenmode calculation for asymmetric slabs (one slab with a radius at the original BIC condition of $R_{0}=$ $0.27 \lambda_{0}$, and the other slab with a radius $\left.R_{1}=R_{0}+3 \delta_{R}\right)$ and varied the gap. From Fig. 10(b) we can see that the asymmetry only shifts the BIC slightly and that tuning of the gap can only offer minimal improvement, we cannot reach the same high $Q$ factors that can be achieved for the symmetric configuration. To confirm that this effect is due to incomplete interference from asymmetry, we have repeated the calculation with no material loss, shown by the black crosses in Fig. 10(b). We can conclude that one can no longer hit the true BIC condition in the asymmetrical system. An explanation for why mirror symmetry in the $z$ direction is required for a BIC is given in Ref. [51] for a single slab and should also apply for our double-slab system. We suspect that one can boost the DPhoC's resistance to asymmetry by choosing single-slab modes that have a larger span in R space; this perhaps could be achieved by overlapping multiple guided-mode resonances, similar to what is done to achieve wide-band reflectors [99].

\section{Effective mass estimation}

We have assumed that the slab moves uniformly but in reality the slab's mechanical modes will have a spatial distribution. This can be taken into account by estimating the effective mass of the slab for a particular mechanical mode. We have performed additional finite element calculations to estimate the effective mass of a tethered trampoline structure with a diameter of $40 \mu \mathrm{m}$. The suspended device has 8 tethers of length $15 \mu \mathrm{m}$, assuming an underetch of $5 \mu \mathrm{m}$ at the support. We find a fundamental mode eigenfrequency of $228.75 \mathrm{kHz}$ (see Fig. 11). The modal mass is $1.16 \mathrm{ng}$ and the effective mass is $8.8 \mathrm{ng}$ assuming a Gaussian beam of waist $10 \mu \mathrm{m}$ incident on the center of the device. The effective mass is calculated by integrating the overlap of the Gaussian beam with the mechanical mode [100]. To take into account the air holes of the structure, we multiply by a correction factor based on the ratio of the photonic crystal's air-hole area and the area of the membrane with no holes.

Using these values for the effective mass and mechanical frequency (along with parameters from set I in Table II) to 


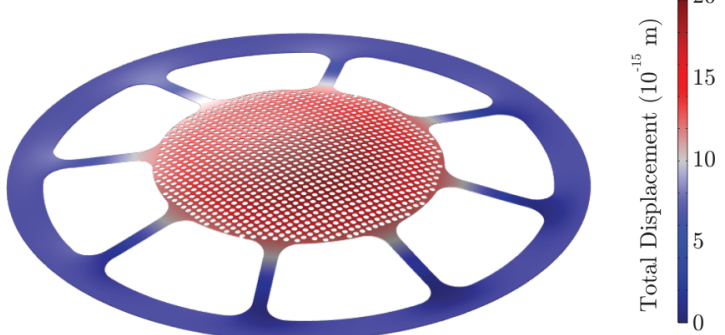

FIG. 11. Spatial profile of the fundamental mechanical mode for a tethered trampoline structure. estimate the optomechanical parameters for the DPhoC gives a single-photon quantum cooperativity of $\sim 0.3$ and falls just short of the ultrastrong single-photon coupling regime with $g_{0} / \Omega_{m} \sim 0.4$. It is likely that with further engineering of the system a value above unity in both cases will be possible. We again emphasize that at no point have any structures been optimized. The effective mass can be reduced to approximately $1 \mathrm{ng}$ by making the device smaller or adding more tethers (which reduces the out-of-plane displacements), but in both cases this will increase the mechanical frequency. Another approach is to decrease the waist of the Gaussian beam, but here one would have to reach a compromise between reducing the effective mass and increased transverse optical losses.
[1] M. Aspelmeyer, T. J. Kippenberg, and F. Marquardt, Cavity optomechanics, Rev. Mod. Phys. 86, 1391 (2014).

[2] J. Chan, T. P. Mayer Alegre, A. H. Safavi-Naeini, J. T. Hill, A. Krause, S. Gröblacher, M. Aspelmeyer, and O. Painter, Laser cooling of a nanomechanical oscillator into its quantum ground state, Nature (London) 478, 89 (2011).

[3] J. D. Teufel, T. Donner, D. Li, J. W. Harlow, M. Allman, K. Cicak, A. J. Sirois, J. D. Whittaker, K. W. Lehnert, and R. W. Simmonds, Sideband cooling of micromechanical motion to the quantum ground state, Nature (London) 475, 359 (2011).

[4] M. Rossi, D. Mason, J. Chen, Y. Tsaturyan, and A. Schliesser, Measurement-based quantum control of mechanical motion, Nature (London) 563, 53 (2018).

[5] U. Delić, M. Reisenbauer, K. Dare, D. Grass, V. Vuletić, N. Kiesel, and M. Aspelmeyer, Cooling of a levitated nanoparticle to the motional quantum ground state, Science $\mathbf{3 6 7}, 892$ (2020).

[6] A. H. Safavi-Naeini, S. Gröblacher, J. T. Hill, J. Chan, M. Aspelmeyer, and O. Painter, Squeezed light from a silicon micromechanical resonator, Nature (London) 500, 185 (2013).

[7] T. P. Purdy, P.-L. Yu, R. Peterson, N. Kampel, and C. Regal, Strong Optomechanical Squeezing of Light, Phys. Rev. X 3, 031012 (2013).

[8] E. E. Wollman, C. U. Lei, A. J. Weinstein, J. Suh, A. Kronwald, F. Marquardt, A. A. Clerk, and K. C. Schwab, Quantum squeezing of motion in a mechanical resonator, Science 349, 952 (2015).

[9] J.-M. Pirkkalainen, E. Damskägg, M. Brandt, F. Massel, and M. A. Sillanpää, Squeezing of Quantum Noise of Motion in a Micromechanical Resonator, Phys. Rev. Lett. 115, 243601 (2015).

[10] F. Lecocq, J. B. Clark, R. W. Simmonds, J. Aumentado, and J. D. Teufel, Quantum Nondemolition Measurement of a Nonclassical State of a Massive Object, Phys. Rev. X 5, 041037 (2015).

[11] T. A. Palomaki, J. D. Teufel, R. W. Simmonds, and K. W. Lehnert, Entangling mechanical motion with microwave fields, Science 342, 710 (2013).

[12] C. F. Ockeloen-Korppi, E. Damskägg, J.-M. Pirkkalainen, M. Asjad, A. A. Clerk, F. Massel, M. J. Woolley, and M. A. Sillanpää, Stabilized entanglement of massive mechanical oscillators, Nature (London) 556, 478 (2018).
[13] R. Riedinger, A. Wallucks, I. Marinković, C. Löschnauer, M. Aspelmeyer, S. Hong, and S. Gröblacher, Remote quantum entanglement between two micromechanical oscillators, Nature (London) 556, 473 (2018).

[14] J. D. Thompson, B. M. Zwickl, A. M. Jayich, F. Marquardt, S. M. Girvin, and J. G. E. Harris, Strong dispersive coupling of a high-finesse cavity to a micromechanical membrane, Nature (London) 452, 72 (2008).

[15] J. C. Sankey, C. Yang, B. M. Zwickl, A. M. Jayich, and J. G. Harris, Strong and tunable nonlinear optomechanical coupling in a low-loss system, Nat. Phys. 6, 707 (2010).

[16] A. A. Clerk, F. Marquardt, and J. G. E. Harris, Quantum Measurement of Phonon Shot Noise, Phys. Rev. Lett. 104, 213603 (2010).

[17] T. P. Purdy, D. Brooks, T. Botter, N. Brahms, Z.-Y. Ma, and D. M. Stamper-Kurn, Tunable Cavity Optomechanics with Ultracold Atoms, Phys. Rev. Lett. 105, 133602 (2010).

[18] M. R. Vanner, Selective Linear or Quadratic Optomechanical Coupling Via Measurement, Phys. Rev. X 1, 021011 (2011).

[19] T. K. Paraïso, M. Kalaee, L. Zang, H. Pfeifer, F. Marquardt, and O. Painter, Position-Squared Coupling in a Tunable Photonic Crystal Optomechanical Cavity, Phys. Rev. X 5, 041024 (2015).

[20] E. Gavartin, R. Braive, I. Sagnes, O. Arcizet, A. Beveratos, T. J. Kippenberg, and I. Robert-Philip, Optomechanical Coupling in a Two-Dimensional Photonic Crystal Defect Cavity, Phys. Rev. Lett. 106, 203902 (2011).

[21] R. Leijssen, G. R. La Gala, L. Freisem, J. T. Muhonen, and E. Verhagen, Nonlinear cavity optomechanics with nanomechanical thermal fluctuations, Nat. Commun. 8, 1 (2017).

[22] J. Guo, R. Norte, and S. Gröblacher, Feedback Cooling of a Room Temperature Mechanical Oscillator Close to Its Motional Ground State, Phys. Rev. Lett. 123, 223602 (2019).

[23] S. Gigan, H. Böhm, M. Paternostro, F. Blaser, G. Langer, J. Hertzberg, K. C. Schwab, D. Bäuerle, M. Aspelmeyer, and A. Zeilinger, Self-cooling of a micromirror by radiation pressure, Nature (London) 444, 67 (2006).

[24] O. Arcizet, P.-F. Cohadon, T. Briant, M. Pinard, and A. Heidmann, Radiation-pressure cooling and optomechanical instability of a micromirror, Nature (London) 444, 71 (2006). 
[25] D. Kleckner and D. Bouwmeester, Sub-kelvin optical cooling of a micromechanical resonator, Nature (London) 444, 75 (2006).

[26] A. Xuereb, C. Genes, and A. Dantan, Strong Coupling and Long-Range Collective Interactions in Optomechanical Arrays, Phys. Rev. Lett. 109, 223601 (2012).

[27] B. Nair, A. Naesby, and A. Dantan, Optomechanical characterization of silicon nitride membrane arrays, Opt. Lett. 42, 1341 (2017).

[28] P. Piergentili, L. Catalini, M. Bawaj, S. Zippilli, N. Malossi, R. Natali, D. Vitali, and G. Di Giuseppe, Two-membrane cavity optomechanics, New J. Phys. 20, 083024 (2018).

[29] C. Gärtner, J. P. Moura, W. Haaxman, R. A. Norte, and S. Gröblacher, Integrated optomechanical arrays of two high reflectivity SiN membranes, Nano Lett. 18, 7171 (2018).

[30] X. Wei, J. Sheng, C. Yang, Y. Wu, and H. Wu, Controllable two-membrane-in-the-middle cavity optomechanical system, Phys. Rev. A 99, 023851 (2019).

[31] A. Xuereb, C. Genes, and A. Dantan, Collectively enhanced optomechanical coupling in periodic arrays of scatterers, Phys. Rev. A 88, 053803 (2013).

[32] S. Fan, W. Suh, and J. D. Joannopoulos, Temporal coupledmode theory for the Fano resonance in optical resonators, J. Opt. Soc. Am. A 20, 569 (2003).

[33] X. Chen, C. Chardin, K. Makles, C. Caër, S. Chua, R. Braive, I. Robert-Philip, T. Briant, P.-F. Cohadon, A. Heidmann et al., High-finesse Fabry-Perot cavities with bidimensional Si3N4 photonic-crystal slabs, Light: Sci. Appl. 6, e16190 (2017).

[34] R. A. Norte, J. P. Moura, and S. Gröblacher, Mechanical Resonators for Quantum Optomechanics Experiments at Room Temperature, Phys. Rev. Lett. 116, 147202 (2016).

[35] S. K. Manjeshwar, K. Elkhouly, J. M. Fitzgerald, M. Ekman, Y. Zhang, F. Zhang, S. M. Wang, P. Tassin, and W. Wieczorek, Suspended photonic crystal membranes in AlGaAs heterostructures for integrated multi-element optomechanics, Appl. Phys. Lett. 116, 264001 (2020).

[36] W. Suh, O. Solgaard, and S. Fan, Displacement sensing using evanescent tunneling between guided resonances in photonic crystal slabs, J. Appl. Phys. 98, 033102 (2005).

[37] Y. Shuai, D. Zhao, Z. Tian, J.-H. Seo, D. V. Plant, Z. Ma, S. Fan, and W. Zhou, Double-layer Fano resonance photonic crystal filters, Opt. Express 21, 24582 (2013).

[38] Y. Liu, W. Zhou, and Y. Sun, Optical refractive index sensing based on high-Q bound states in the continuum in free-space coupled photonic crystal slabs, Sensors 17, 1861 (2017).

[39] U. Kemiktarak, M. Metcalfe, M. Durand, and J. Lawall, Mechanically compliant grating reflectors for optomechanics, Appl. Phys. Lett. 100, 061124 (2012).

[40] D. Woolf, P.-C. Hui, E. Iwase, M. Khan, A. W. Rodriguez, P. Deotare, I. Bulu, S. G. Johnson, F. Capasso, and M. Loncar, Optomechanical and photothermal interactions in suspended photonic crystal membranes, Opt. Express 21, 7258 (2013).

[41] K. Makles, T. Antoni, A. Kuhn, S. Deléglise, T. Briant, P.-F. Cohadon, R. Braive, G. Beaudoin, L. Pinard, C. Michel et al., $2 \mathrm{D}$ photonic-crystal optomechanical nanoresonator, Opt. Lett. 40, 174 (2015).

[42] C. Stambaugh, H. Xu, U. Kemiktarak, J. Taylor, and J. Lawall, From membrane-in-the-middle to mirror-in-the-middle with a high-reflectivity sub-wavelength grating, Ann. Phys. (Berlin) 527, 81 (2015).

[43] Y.-G. Roh, T. Tanabe, A. Shinya, H. Taniyama, E. Kuramochi, S. Matsuo, T. Sato, and M. Notomi, Strong optomechanical interaction in a bilayer photonic crystal, Phys. Rev. B 81, 121101(R) (2010).

[44] A. Naesby and A. Dantan, Microcavities with suspended subwavelength structured mirrors, Opt. Express 26, 29886 (2018).

[45] O. Černotík, A. Dantan, and C. Genes, Cavity Quantum Electrodynamics with Frequency-Dependent Reflectors, Phys. Rev. Lett. 122, 243601 (2019).

[46] D. Marinica, A. Borisov, and S. Shabanov, Bound States in the Continuum in Photonics, Phys. Rev. Lett. 100, 183902 (2008).

[47] C. W. Hsu, B. Zhen, A. D. Stone, J. D. Joannopoulos, and M. Soljačić, Bound states in the continuum, Nat. Rev. Mater. 1, 1 (2016).

[48] B. Guha, S. Mariani, A. Lemaître, S. Combrié, G. Leo, and I. Favero, High frequency optomechanical disk resonators in III-V ternary semiconductors, Opt. Express 25, 24639 (2017).

[49] S. Bernard, C. Reinhardt, V. Dumont, Y.-A. Peter, and J. C. Sankey, Precision resonance tuning and design of SiN photonic crystal reflectors, Opt. Lett. 41, 5624 (2016).

[50] H. A. Haus, Waves and Fields in Optoelectronics (PrenticeHall, Englewodd Cliffs, NJ, 1984).

[51] C. W. Hsu, B. Zhen, J. Lee, S.-L. Chua, S. G. Johnson, J. D. Joannopoulos, and M. Soljačić, Observation of trapped light within the radiation continuum, Nature (London) 499, 188 (2013).

[52] R. Gansch, S. Kalchmair, P. Genevet, T. Zederbauer, H. Detz, A. M. Andrews, W. Schrenk, F. Capasso, M. Lončar, and G. Strasser, Measurement of bound states in the continuum by a detector embedded in a photonic crystal, Light: Sci. Appl. 5, e16147 (2016).

[53] A. Kodigala, T. Lepetit, Q. Gu, B. Bahari, Y. Fainman, and B. Kanté, Lasing action from photonic bound states in continuum, Nature (London) 541, 196 (2017).

[54] L. Li and H. Yin, Bound states in the continuum in double layer structures, Sci. Rep. 6, 26988 (2016).

[55] J. Jin, X. Yin, L. Ni, M. Soljačić, B. Zhen, and C. Peng, Topologically enabled ultrahigh-Q guided resonances robust to out-of-plane scattering, Nature (London) 574, 501 (2019).

[56] M. Zhao and K. Fang, Mechanical bound states in the continuum for macroscopic optomechanics, Opt. Express 27, 10138 (2019).

[57] C. B. R. Hurtado, J. Dickmann, F. F. Bruns, T. Siefke, and S. Kroker, Bound states in the continuum for optomechanical light control with dielectric metasurfaces, Opt. Express 28, 20106 (2020).

[58] D. Van Thourhout and J. Roels, Optomechanical device actuation through the optical gradient force, Nat. Photonics 4, 211 (2010).

[59] M. Eichenfield, C. P. Michael, R. Perahia, and O. Painter, Actuation of micro-optomechanical systems via cavity-enhanced optical dipole forces, Nat. Photonics 1, 416 (2007).

[60] G. Anetsberger, O. Arcizet, Q. P. Unterreithmeier, R. Rivière, A. Schliesser, E. M. Weig, J. P. Kotthaus, and T. J. Kippenberg, Near-field cavity optomechanics with nanomechanical oscillators, Nat. Phys. 5, 909 (2009). 
[61] G. S. Wiederhecker, L. Chen, A. Gondarenko, and M. Lipson, Controlling photonic structures using optical forces, Nature (London) 462, 633 (2009).

[62] C. Michael, K. Srinivasan, T. Johnson, O. Painter, K. Lee, K. Hennessy, H. Kim, and E. Hu, Wavelength-and materialdependent absorption in GaAs and AlGaAs microcavities, Appl. Phys. Lett. 90, 051108 (2007).

[63] P. Rabl, Photon Blockade Effect in Optomechanical Systems, Phys. Rev. Lett. 107, 063601 (2011).

[64] A. Nunnenkamp, K. Børkje, and S. M. Girvin, Single-Photon Optomechanics, Phys. Rev. Lett. 107, 063602 (2011).

[65] F. Fogliano, B. Besga, A. Reigue, P. Heringlake, L. M. de Lépinay, C. Vaneph, J. Reichel, B. Pigeau, and O. Arcizet, Cavity nano-optomechanics in the ultrastrong coupling regime with ultrasensitive force sensors, arXiv:1904.01140.

[66] Y. Tsaturyan, A. Barg, E. S. Polzik, and A. Schliesser, Ultracoherent nanomechanical resonators via soft clamping and dissipation dilution, Nat. Nanotechnol. 12, 776 (2017).

[67] K. Børkje, Critical quantum fluctuations and photon antibunching in optomechanical systems with large single-photon cooperativity, Phys. Rev. A 101, 053833 (2020).

[68] G. Via, G. Kirchmair, and O. Romero-Isart, Strong SinglePhoton Coupling in Superconducting Quantum Magnetomechanics, Phys. Rev. Lett. 114, 143602 (2015).

[69] E. Romero-Sanchez, W. P. Bowen, M. R. Vanner, K. Xia, and J. Twamley, Quantum magnetomechanics: Towards the ultrastrong coupling regime, Phys. Rev. B 97, 024109 (2018).

[70] K. W. Murch, K. L. Moore, S. Gupta, and D. M. StamperKurn, Observation of quantum-measurement backaction with an ultracold atomic gas, Nat. Phys. 4, 561 (2008).

[71] F. Brennecke, S. Ritter, T. Donner, and T. Esslinger, Cavity optomechanics with a Bose-Einstein condensate, Science 322, 235 (2008).

[72] F. Elste, S. M. Girvin, and A. A. Clerk, Quantum Noise Interference and Backaction Cooling in Cavity Nanomechanics, Phys. Rev. Lett. 102, 207209 (2009).

[73] A. Xuereb, R. Schnabel, and K. Hammerer, Dissipative Optomechanics in a Michelson-Sagnac Interferometer, Phys. Rev. Lett. 107, 213604 (2011).

[74] M. Wu, A. C. Hryciw, C. Healey, D. P. Lake, H. Jayakumar, M. R. Freeman, J. P. Davis, and P. E. Barclay, Dissipative and Dispersive Optomechanics in a Nanocavity Torque Sensor, Phys. Rev. X 4, 021052 (2014).

[75] D. Wilson, V. Sudhir, N. Piro, R. Schilling, A. Ghadimi, and T. J. Kippenberg, Measurement-based control of a mechanical oscillator at its thermal decoherence rate, Nature (London) 524, 325 (2015).

[76] C. Gut, K. Winkler, J. Hoelscher-Obermaier, S. Hofer, R. M. Nia, N. Walk, A. Steffens, J. Eisert, W. Wieczorek, J. Slater et al., Stationary optomechanical entanglement between a mechanical oscillator and its measurement apparatus, Phys. Rev. Research 2, 033244 (2020).

[77] H.-K. Lau and A. A. Clerk, Ground-State Cooling and HighFidelity Quantum Transduction Via Parametrically Driven Bad-Cavity Optomechanics, Phys. Rev. Lett. 124, 103602 (2020).

[78] M. R. Vanner, I. Pikovski, G. D. Cole, M. Kim, Č. Brukner, K. Hammerer, G. J. Milburn, and M. Aspelmeyer, Pulsed quantum optomechanics, Proc. Natl. Acad. Sci. U. S. A. 108, 16182 (2011).
[79] J. Clarke, P. Sahium, K. E. Khosla, I. Pikovski, M. S. Kim, and M. R. Vanner, Generating mechanical and optomechanical entanglement via pulsed interaction and measurement, New J. Phys. 22, 063001 (2020).

[80] M. Notomi, H. Taniyama, S. Mitsugi, and E. Kuramochi, Optomechanical Wavelength and Energy Conversion in high-Q Double-Layer Cavities of Photonic Crystal Slabs, Phys. Rev. Lett. 97, 023903 (2006).

[81] V. Liu and S. Fan, $\mathrm{S}^{4}$ : A free electromagnetic solver for layered periodic structures, Comput. Phys. Commun. 183, 2233 (2012).

[82] W. Suh, M. F. Yanik, O. Solgaard, and S. Fan, Displacementsensitive photonic crystal structures based on guided resonance in photonic crystal slabs, Appl. Phys. Lett. 82, 1999 (2003).

[83] H. Y. Song, S. Kim, and R. Magnusson, Tunable guidedmode resonances in coupled gratings, Opt. Express 17, 23544 (2009).

[84] C. Blanchard, J.-P. Hugonin, and C. Sauvan, Fano resonances in photonic crystal slabs near optical bound states in the continuum, Phys. Rev. B 94, 155303 (2016).

[85] J. M. Llorens, J. Buencuerpo, and P. A. Postigo, Absorption features of the zero frequency mode in an ultra-thin slab, Appl. Phys. Lett. 105, 231115 (2014).

[86] N. Ismail, C. C. Kores, D. Geskus, and M. Pollnau, FabryPérot resonator: Spectral line shapes, generic and related airy distributions, linewidths, finesses, and performance at low or frequency-dependent reflectivity, Opt. Express 24, 16366 (2016).

[87] G. Wachter, S. Kuhn, S. Minniberger, C. Salter, P. Asenbaum, J. Millen, M. Schneider, J. Schalko, U. Schmid, A. Felgner et al., Silicon microcavity arrays with open access and a finesse of half a million, Light: Sci. Appl. 8, 1 (2019).

[88] S. Gröblacher, J. B. Hertzberg, M. R. Vanner, G. D. Cole, S. Gigan, K. Schwab, and M. Aspelmeyer, Demonstration of an ultracold micro-optomechanical oscillator in a cryogenic cavity, Nat. Phys. 5, 485 (2009).

[89] G. D. Cole, I. Wilson-Rae, K. Werbach, M. R. Vanner, and M. Aspelmeyer, Phonon-tunnelling dissipation in mechanical resonators, Nat. Commun. 2, 1 (2011).

[90] M. J. Weaver, B. Pepper, F. Luna, F. M. Buters, H. J. Eerkens, G. Welker, B. Perock, K. Heeck, S. de Man, and D. Bouwmeester, Nested trampoline resonators for optomechanics, Appl. Phys. Lett. 108, 033501 (2016).

[91] T. Xu, M. S. Wheeler, H. E. Ruda, M. Mojahedi, and J. S. Aitchison, The influence of material absorption on the quality factor of photonic crystal cavities, Opt. Express 17, 8343 (2009).

[92] J. Lee, B. Zhen, S.-L. Chua, O. Shapira, and M. Soljačić, Fabricating centimeter-scale high quality factor two-dimensional periodic photonic crystal slabs, Opt. Express 22, 3724 (2014).

[93] D. Fattal, J. Li, Z. Peng, M. Fiorentino, and R. G. Beausoleil, Flat dielectric grating reflectors with focusing abilities, Nat. Photonics 4, 466 (2010).

[94] J. Guo, R. A. Norte, and S. Gröblacher, Integrated optical force sensors using focusing photonic crystal arrays, Opt. Express 25, 9196 (2017).

[95] J. P. Moura, R. A. Norte, J. Guo, C. Schäfermeier, and S. Gröblacher, Centimeter-scale suspended photonic crystal mirrors, Opt. Express 26, 1895 (2018). 
[96] A. Taghizadeh and I.-S. Chung, Quasi bound states in the continuum with few unit cells of photonic crystal slab, Appl. Phys. Lett. 111, 031114 (2017).

[97] E. Serra, M. Bawaj, A. Borrielli, G. Di Giuseppe, S. Forte, N. Kralj, N. Malossi, L. Marconi, F. Marin, F. Marino et al., Microfabrication of large-area circular high-stress silicon nitride membranes for optomechanical applications, AIP Adv. 6, 065004 (2016).
[98] S. Adachi, GaAs, AlAs, and $\mathrm{Al}_{x} \mathrm{Ga}_{1-x}$ As: Material parameters for use in research and device applications, J. Appl. Phys. 58, R1 (1985).

[99] R. Magnusson and M. Shokooh-Saremi, Physical basis for wideband resonant reflectors, Opt. Express 16, 3456 (2008).

[100] M. Pinard, Y. Hadjar, and A. Heidmann, Effective mass in quantum effects of radiation pressure, Eur. Phys. J. D 7, 107 (1999). 